in Proc. IEEE SSP-03, St. Louis (MO), Sept./Oct. 2003, pp. 621-624

Copyright IEEE 2003

\title{
STABLE ADAPTIVE NONSTATIONARY SIGNAL DETECTION BASED ON THE ROBUSTON SCHEME*
}

\author{
Michael Jachan, Franz Hlawatsch, and Gerald Matz
}

Institute of Communications and Radio-Frequency Engineering, Vienna University of Technology

Gusshausstrasse 25/389, A-1040 Vienna, Austria

Tel.: +43158801 38914, Fax: +43158801 38999, E-mail: michael.jachan@tuwien.ac.at

web: http://www.nt.tuwien.ac.at/dspgroup/time.html

\begin{abstract}
The robuston scheme is a novel reduced-detail paradigm for nonstationary signal modeling/processing with enhanced statistical stability. Here, we apply the robuston scheme to the problem of detecting a nonstationary random signal in white Gaussian noise. We propose two different "robuston detectors" along with signal-adaptive online implementations that perform online estimation of the signal statistics from a single observation. The performance of adaptive robuston detectors is assessed through numerical simulations.
\end{abstract}

\section{INTRODUCTION}

In nonstationary environments, statistical signal models that are very detailed may not allow reliable estimation of the statistics involved. We therefore introduced the robuston scheme as a reduceddetail paradigm for nonstationary signal modeling/processing with enhanced statistical stability, and we applied this scheme to signal estimation [1]. Robuston signal modeling/processing uses timefrequency localized subspace signal components (called robustons) as atomic entities; it employs special time-varying filters (robuston filters) that allow an efficient online implementation, and statistical signal descriptors (robuston correlations) that can be estimated in a stable manner by means of intra-subspace averaging [1].

In this paper, we apply the robuston scheme to the problem of detecting a nonstationary random signal in white Gaussian noise. We introduce two types of "robuston detectors": the robuston likelihood-ratio detector (RLD) for a Gaussian signal process and the robuston deflection-optimal detector (RDD) for a non-Gaussian signal process. For practical application, we also propose signal-adaptive versions of these robuston detectors that perform online estimation of the signal statistics (i.e., robuston correlations) from a single observation. The RLD and RDD generalize minimax-robust detectors previously introduced in [2].

This paper is organized as follows. After a review of the robuston scheme in Section 2, the RLD and RDD are introduced in Section 3. In Section 4, adaptive implementations of these detectors are proposed. Finally, Section 5 presents simulation results assessing the performance of adaptive robuston detectors.

\section{REVIEW OF THE ROBUSTON SCHEME}

Robuston signal decomposition. The robuston scheme is based on a decomposition of a discrete-time signal $x[n]$ into orthogonal subspace components (robustons) $x_{k, I}[n]$, i.e., $x[n]=\sum_{k=-\infty}^{\infty} \sum_{l=0}^{L-1} x_{k, l}[n]$ $[1-4]$. The robustons are used as atomic entities for statistical signal modeling and processing. The $(k, l)$ th robuston $x_{k, l}[n]$ lies in a $P$-dimensional linear signal subspace $\mathscr{X}_{k . l}$. As shown in Fig. 1 , it is effectively located in a time-frequency rectangle corresponding to the time interval (block) $[k N,(k+1) N]$ with block length $N=L P$ and the frequency band $\left[\frac{l}{2 L}, \frac{l+1}{2 L}\right]=\left[\frac{l P}{2 N}, \frac{(l+1) P}{2 N}\right]$ with bandwidth $1 /(2 L)=P /(2 N)$. There are $L$ frequency bands.

*Funding by FWF grant P15156-N02.

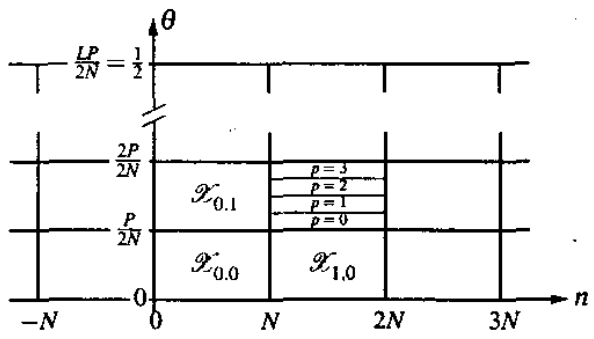

Figure 1: Time-frequency location of the robustons $x_{k, j}[n]$. The subspace $\mathscr{X}_{1,1}$ is blown up into its LCB functions (subspace dimension $P=4$ assimed). Only positive frequencies are shown.

The robuston subspace $\mathscr{X}_{k, l}$ containing the robuston $x_{k, l}[n]$ is spanned by the $P$ orthonormal local cosine basis $(L C B)$ functions [5] $u_{k . l}^{(p)}[n]=\sqrt{\frac{2}{N}} \cos \left(\pi \frac{\mid P+p+1 / 2}{N}(n-k N)\right) w[n-k N]$ with $p \in$ $\{0,1, \cdots, P-1\}$. Here, $w^{\prime}[\hat{n}]$ is a suitably chosen window with effective time duration $N$ [5], and $p$ is an "intra-band" frequency index (within the $l$ th frequency band). Thus ${ }^{1}$,

$$
x_{k, l}[n]=\sum_{p=0}^{P-1} \alpha_{k, l}^{(p)} u_{k, l}^{(p)}[n] \in \mathscr{X}_{k, l} \quad \text { with } \alpha_{k, l}^{(p)}=\left\langle x, u_{k, l}^{(p)}\right\rangle
$$

Efficient discrete cosine transform (DCT) algorithms for LCB analysis and synthesis are available $[5,6]$.

Robuston filters. Robuston signal processing is performed by robuston filters (RFs). [1]. An RF $\mathbb{H}$ is a linear, time-varying system such that each robuston $y_{k, l}[n]$ of the filter output $y[n]=(\mathbb{H} x)[n]$ is a weighted sum of (temporally adjacent) robustons $x_{k^{\prime}, l}[n]$ of the filter input $x[n]$ in the same (lth) frequency band:

$$
y_{k, l}[n]=\sum_{k^{\prime}=k-M_{1}}^{k+M_{2}} h_{k, k^{\prime}, l} x_{k^{\prime}, l}\left[n-\left(k-k^{\prime}\right) N\right]=\mathbf{h}_{k, l}^{T} \mathbf{x}_{k, l}[n],
$$

with the vectors $\mathbf{h}_{k, l}=\left[h_{k, k-M_{1} l} \cdots h_{k, k+M_{2}, l}\right]^{T}$ and $\mathbf{x}_{k, l}[n]=$ $\left[x_{k-M_{1}, l}\left[n-M_{1} N\right] \cdots x_{k+M_{2}, l}\left[n+M_{2} N\right]\right]^{T}$ of length $M=M_{1}+M_{2}+$ 1. Note that there is no filtering across frequency bands. $M_{1}$ and $M_{2}$ are the anticausal and causal filter lengths, respectively.

It can be shown that an RF can be written as

$$
\mathbb{H}=\sum_{k=-\infty}^{\infty} \sum_{l=0}^{L-1} \mathbb{H}_{k, l} \quad \text { with } \mathbb{H}_{k, l}=\sum_{k=k-M_{1}}^{k+M_{2}} h_{k, k^{\prime} ! l} \mathbb{P}_{k, k^{\prime}: l},
$$

with the "shift-projector" $\mathbb{P}_{k, k^{\prime}, l} \triangleq \mathbb{S}_{k-k^{\prime}} \mathbb{P}_{k^{\prime}, l}=\mathbb{P}_{k, l} \mathbb{S}_{k-k^{\prime}}$ [1]. Here, $\mathbb{P}_{k, l}$ is the orthogonal projector on $\mathscr{X}_{k, l}$ (i.e., $\left.x_{k, l}[n]=\left(\mathbb{P}_{k, l} x\right)[n]\right)$

\footnotetext{
${ }^{1}$ The signal inner product is defined as $\langle x, y\rangle \triangleq \sum_{n=-\infty}^{\infty} x[n] y^{*}[n]$.
} 
and $\mathbb{S}_{k}$ is the block time shift operator (i.e., $\left(\mathbb{S}_{k} x\right)[n]=x[n-k N]$ ). The kernel of $\mathbb{P}_{k, k^{\prime} ; l}$ is given by $p_{k, k^{\prime} ; l}\left[n, n^{\prime}\right]=\sum_{p=0}^{P-1} u_{k, l}^{(p)}[n] u_{k^{\prime}, l}^{(p) *}\left[n^{\prime}\right]$.

Robuston correlations. We next consider a zero-mean nonstationary random process $x[n]$ with correlation operator $\mathbb{R}$ (the linear operator whose kernel is $R\left[n, n^{\prime}\right]=\mathrm{E}\left\{x[n] x^{*}\left[n^{\prime}\right]\right\}$ ). A complete description of the second-order statistics of $x[n]$ would generally involve all LCB coefficient correlations $\mathrm{E}\left\{\alpha_{k, l}^{(p)} \alpha_{k^{\prime}, l^{\prime}}^{\left(p^{\prime}\right) *}\right\}$. In contrast, the robuston desciption of $x[n]$ corresponds to a reduced-detail model that uses only the robuston correlations (RCs) [1]

$$
r_{k, k^{\prime}, l} \triangleq \frac{1}{P} \sum_{p=0}^{P-1} \mathrm{E}\left\{\alpha_{k . l}^{(p)} \alpha_{k^{\prime}, l}^{(p) *}\right\}=\frac{1}{P} \mathrm{E}\left\{\left\langle x_{k, l}, \mathbb{S}_{k-k^{\prime}} x_{k^{\prime}, l}\right\rangle\right\}
$$

These (mathematically equivalent) expressions show that the RC $r_{k, k^{\prime} ; l}$ is (i) the average of $\mathrm{E}\left\{\alpha_{k . l}^{(p)} \alpha_{k^{\prime}, l}^{(p) *}\right\}$ over all intra-band frequencies $p=0, \cdots, P-1$, and (ii) an integral measure of the correlation of the two robustons $x_{k, l}[n]$ and $x_{k, l}[n]$ located in the $k$ th and $k^{\prime}$ th time blocks and the $l$ th frequency band (thus, no correlations across frequency bands are taken into account).

An unbiased estimator of $r_{k, k^{\prime} ; l}$ using a single realization of $x[n]$ is given by (cf. (3))

$$
\hat{r}_{k, k^{\prime}, l}=\frac{1}{P} \sum_{p=0}^{P-1} \alpha_{k, l}^{(p)} \alpha_{k^{\prime}, l}^{(p) *}=\frac{1}{P}\left\langle x_{k, l}, \mathbb{S}_{k-k^{\prime}}, x_{k^{\prime}, l}\right\rangle=\frac{1}{P}\left\langle x, \mathbb{P}_{k, k^{\prime} ; l} x\right\rangle \text {. }
$$

Note the averaging over all intra-band frequencies $p=0, \cdots, P-$ 1 corresponding to $P$ orthogonal LCB components. This intrasubspace averaging results in enhanced statistical stability of $\hat{r}_{k, k^{\prime}, l}$.

For later use, we introduce the robuston correlation operator $\mathbb{R}^{\mathrm{R}} \triangleq \sum_{k=-\infty}^{\infty} \sum_{l=0}^{L-1} \sum_{k^{\prime}=k^{-}-M_{1}}^{k+M_{2}} r_{k \cdot k^{\prime}: l} \mathbb{P}_{k, k^{\prime}: l}$. A process $x[n]$ will be called a robuston process (RP) if $\mathbb{R}=\mathbb{R}^{R}$. Thus, for an $R P$, the $\mathrm{RCs} r_{k, k^{\prime} ; l}$ provide a complete second-order description.

\section{ROBUSTON DETECTORS}

We now consider the application of robuston signal modeling/processing to the problem of detecting a nonstationary, zero-mean random signal $s[n]$ in white Gaussian noise $z[n]$ that is statistically independent of $s[n]$. We assume that the noise variance $\sigma_{z}^{2}$ is known. Our goal is to discriminate between the hypotheses $\mathscr{H}_{0}: x[n]=z[n]$ and $\mathscr{H}_{1}: x[n]=s[n]+z[n]$, where $x[n]$ is the observed signal [7]. Initially, we assume that the RCs of $s[n]$ are known. Later (in Section 4), these RCs will be replaced by estimates.

\subsection{The Robuston Likelihood-Ratio Detector}

We first assume that the signal process $s[n]$ is Gaussian. The classical likelihood-ratio test statistic (which is optimal both in the Neyman-Pearson sense and in the Bayesian sense) is given by [7]

$$
T_{\mathrm{L}}(x)=\left\langle\mathbb{H}_{\mathrm{L}} x, x\right\rangle \quad \text { with } \mathbb{H}_{\mathrm{L}}=\frac{1}{\sigma_{z}^{2}} \mathbb{R}_{s}\left(\mathbb{R}_{5}+\sigma_{z}^{2} \mathbb{I}\right)^{-1},
$$

where $\mathbb{R}_{s}$ is the correlation operator of $s[n]$ and $\mathbb{I}$ is the identity operator. The likelihood-ratio detector is closely related to the Wiener filter $\mathbb{H}_{\mathrm{W}}=\mathbb{R}_{s}\left(\mathbb{R}_{s}+\sigma_{z}^{2} \mathbb{I}\right)^{-1}$ because $\mathbb{H}_{\mathrm{L}}=\frac{1}{\sigma^{2}} \mathbb{H}_{\mathrm{W}}$. The Wiener filter is the linear time-varying (LTV) filter that estimates $s[n]$ from $x[n]=s[n]+z[n]$ with minimum mean-square error (MSE) [7].

Building on this "estimation connection," we can develop a robuston variant of the likelihood-ratio detector as follows. In [1], the robuston Wiener filter $\widetilde{\mathrm{H}}_{\mathrm{W}}$ was introduced as the RF signal estimator with minimum MSE. For given filter lengths $M_{1}, M_{2}$, the coefficient vectors (cf. (1)) of the RF. $\tilde{\mathbb{H}}_{\mathrm{W}}$ are given by [1]

$$
\mathbf{h}_{k, l}^{\mathrm{W}}=\mathbf{R}_{k, l}^{-1} \mathbf{r}_{k, l}^{(s)}=\left(\mathbf{R}_{k, l}^{(s)}+\sigma_{z}^{2} \mathbf{I}\right)^{-1} \mathbf{r}_{k, l}^{(s)},
$$

with the RC matrices $\left[\mathbf{R}_{k . l}\right]_{m \cdot m^{\prime}}=r_{k-M_{1}+m^{\prime}-1, k-M_{1}+m-1: l}$ and $\left[\mathbf{R}_{k . l}^{(s)}\right]_{m, m^{\prime}}=r_{k-M_{1}+m^{\prime}-1 . k-M_{1}+m-1 ; l}^{(s)}$ and the signal $\mathrm{RC}$ vector $\mathbf{r}_{k . l}^{(s)}=\left[r_{k . k-M_{1} ;}^{(s)} \cdots r_{k, k+M_{2} ; l}^{(s)}\right]^{T}$ (here, $m, m^{\prime} \in\{1, \ldots, M\}$ with the overall filter length $M=M_{1}+M_{2}+1$, and $r_{k, k^{\prime} ; l}$ and $r_{k, k^{\prime} ; l}^{(s)}$ denote the RCs of $x[n]$ and $s[n]$, respectively). We now introduce the robuston likelihood-ratio detector (RLD) by replacing in the likelihood ratio test statistic (5) the Wiener filter $\mathbb{H}_{\mathrm{W}}$ with the robuston Wiener filter $\tilde{\mathbb{H}}_{\mathrm{W}}$ (with given parameters $N, P, M_{1}$, and $M_{2}$ ). This yields the RLD test statistic as

$$
\tilde{T}_{\mathrm{L}}(x) \triangleq\left\langle\overline{\mathbb{H}}_{\mathrm{L}} x, x\right\rangle \quad \text { with } \tilde{\mathbb{H}}_{\mathrm{L}}=\frac{1}{\sigma_{z}^{2}} \tilde{\mathbb{H}}_{\mathrm{W}} .
$$

The RLD can be formulated and implemented as follows. According to (2), $\tilde{\mathbb{H}}_{\mathrm{L}}=\sum_{k=-\infty}^{\infty} \sum_{l=0}^{L-1} \sum_{k^{\prime}=k-M_{1}}^{k+M_{2}} \tilde{h}_{k, k^{\prime} ; l}^{\mathrm{L}} \mathbb{P}_{k, k^{\prime} ; l^{\prime}}$. Inserting this into (7) and using (4), we obtain

$$
\begin{aligned}
\tilde{T}_{\mathrm{L}}(x) & =\sum_{k=-\infty}^{\infty} \sum_{l=0}^{L-1} \sum_{k^{\prime}=k-M_{1}}^{k+M_{2}} \tilde{h}_{k, k^{\prime} ; l}^{\mathrm{L}}\left\langle\mathbb{P}_{k, k^{\prime} ; l} x, x\right\rangle \\
& =P \sum_{k=-\infty}^{\infty} \sum_{l=0}^{L-1} \sum_{k^{\prime}=k-M_{1}}^{k+M_{2}} \tilde{h}_{k, k^{\prime} ;}^{\mathrm{L}} \hat{r}_{k, k^{\prime} ; l}^{*}=P \sum_{k=-\infty}^{\infty} \sum_{l=0}^{L-1} \hat{\mathbf{r}}_{k, l}^{H} \mathbf{h}_{k, l}^{\mathrm{L}} .
\end{aligned}
$$

Here, $\hat{\mathbf{r}}_{k, l}=\left[\hat{r}_{k, k-M_{1}: l} \cdots \hat{r}_{k, k+M_{2}, l}\right]^{T}$ with $\hat{r}_{k, k^{\prime} ; l}$ defined in (4), and $\mathbf{h}_{k, l}^{\mathrm{L}}=\left[\tilde{h}_{k, k-M_{1}: l}^{\mathrm{L}} \cdots \tilde{h}_{k, k+M_{2}: l}^{\mathrm{L}}\right]^{T}$ is given by (cf. (6))

$$
\mathbf{h}_{k . l}^{\mathrm{L}}=\frac{1}{\sigma_{z}^{2}} \mathbf{h}_{k . l}^{\mathrm{W}}=\frac{1}{\sigma_{z}^{2}}\left(\mathbf{R}_{k . l}^{(s)}+\sigma_{z}^{2} \mathrm{I}\right)^{-1} \mathbf{r}_{k . l}^{(s)} .
$$

It is important to note that the RLD only presupposes know]edge of the RCs of $s[n]$; knowledge of the complete second-order statistics (correlation operator $\mathbb{R}_{s}$ ) is not required. Furthermore, the operator multiplication and inversion required for computing $\mathbb{H}_{\mathrm{L}}=\frac{1}{\sigma_{2}^{2}} \mathbb{R}_{s}\left(\mathbb{R}_{\mathrm{s}}+\sigma_{z}^{2} \mathbb{I}\right)^{-1}$ (note that these operators correspond to matrices whose size equals the total signal length) is replaced by matrix inversions and matrix-vector multiplications of size $M \times M$, which is typically small. If $s[\boldsymbol{n}]$ is an RP, the classical likelihoodratio detector in (5) reduces to the RLD (with $M_{1}, M_{2}$ generally infinite however), i.e., $\mathbb{H}_{\mathrm{L}}=\mathbb{H}_{\mathrm{L}}$ and $T_{\mathrm{L}}(x)=\tilde{T}_{\mathrm{L}}(x)$. Hence, the robuston structure of $s[n]$ enforces a robuston structure on $\mathbb{H}_{L}$.

\subsection{The Robuston Deflection-Optimal Detector}

If the signal process $s[n]$ is not Gaussian, the likelihood-ratio detector often is hard or impossible to calculate. A practical alternative is provided by the deflection-optimal detector that maximizes a contrast criterion known as deflection and defined as $[7,8]$

$$
d^{2} \triangleq \frac{\left(\mathrm{E}\left\{T(x) \mid \mathscr{H}_{1}\right\}-\mathrm{E}\left\{T(x) \mid \mathscr{H}_{0}\right\}\right)^{2}}{\operatorname{var}\left\{T(x) \mid \mathscr{H}_{0}\right\}} .
$$

The (quadratic) deflection-optimal detector is obtained as [8]

$$
T_{\mathrm{D}}(x)=\left\langle\mathbb{H}_{\mathrm{D}} x, x\right\rangle \quad \text { with } \mathbb{H}_{\mathrm{D}}=\frac{1}{\sigma_{z}^{4}} \mathbb{R}_{s} .
$$

We now define the robuston deflection-optimal detector (RDD) as the quadratic test statistic induced by the $\mathrm{RF} \tilde{\mathbb{H}}_{\mathrm{D}}$ (with given parameters $N, P, M_{1}, M_{2}$ ) that maximizes the deflection:

$$
\tilde{T}_{\mathrm{D}}(x)=\left\langle\tilde{\mathbb{H}}_{\mathrm{D}} x, x\right\rangle \quad \text { with } \tilde{\mathbb{H}}_{\mathrm{D}} \triangleq \arg \max _{\tilde{\mathbf{H}} \in \mathscr{R}} d^{2},
$$

where $\mathscr{R}$ denotes the set of all RFs (2) with fixed $N, P, M_{1}$, and $M_{2}$.

The RDD can be derived by using the Hilbert space structure of RFs. Indeed, it can be shown that for $M_{1}, M_{2}$ finite, $\mathscr{R}$ is a linear subspace of the Hilbert space $\mathscr{L}$ of all LTV systems (linear oper- 
ators) $\mathbb{H}$ with finite Hilbert-Schmidt norm ${ }^{2}$. The normalized shiftprojectors $\overline{\mathbb{P}}_{k, k^{\prime}: l} \triangleq \frac{1}{\sqrt{P}} \mathbb{P}_{k \cdot k^{\prime} ; l}$ form an orthonormal basis for $\mathscr{R}$. Any $\mathbb{H} \in \mathscr{L}$ allows the orthogonal decomposition $\mathbb{H}=\mathbb{H}^{\mathrm{R}}+\mathbb{H}^{\perp}$, where the RF $\mathbb{H}^{\mathrm{R}}=\sum_{k=-\infty}^{\infty} \sum_{l=0}^{L-1} \sum_{k^{\prime}=k-M_{1}}^{\mathrm{k}+M_{2}} h_{k, k^{\prime} ; l}^{\mathrm{R}} \mathbb{P}_{k, k^{\prime} ; l}$ with coefficients $h_{k, k^{\prime}: l}^{\mathrm{R}}=\frac{1}{P}\left\langle\mathbb{H}, \mathbb{P}_{k, k^{\prime}, l}\right\rangle_{\mathrm{HS}}$ is the orthogonal projection of $\mathbb{H}$ onto $\mathscr{R}$ and $\mathbb{H}^{\perp}$ belongs to the orthogonal complement space $\mathscr{R}^{\perp}$.

It is easily shown [8] that the deflection for any (Hermitian) operator $\tilde{H}$ can be written as

$$
d^{2}=\frac{1}{\sigma_{z}^{4}} \frac{\left\langle\tilde{\mathbb{H}}, \mathbb{R}_{S}\right\rangle_{\mathrm{HS}}^{2}}{\|\tilde{\mathbb{H}}\|_{\mathrm{HS}}^{2}} .
$$

In our case, $\tilde{\mathbb{H}} \in \mathscr{R}$ by assumption and thus $\left\langle\overline{\mathbb{H}}, \mathbb{R}_{s}\right\rangle_{\mathrm{HS}}=\left\langle\tilde{\mathbb{H}}, \mathbb{R}_{s}^{\mathbf{R}}+\right.$ $\left.\mathbb{R}_{s}^{\perp}\right\rangle_{\mathrm{HS}}=\left\langle\check{\mathbb{H}}, \mathbb{R}_{s}^{R}\right\rangle_{\mathrm{HS}}$ because $\mathbb{R}_{s}^{\perp}$ is orthogonal to $\overline{\mathbb{H}}$. We now use the Schwarz inequality [9]: $\left\langle\overline{\mathbb{H}}, \mathbb{R}_{s}^{R}\right\rangle_{\mathrm{HS}}^{2} \leq\|\tilde{\mathbb{H}}\|_{\mathrm{HS}}^{2}\left\|\mathbb{R}_{s}^{\mathrm{R}}\right\|_{\mathrm{HS}}^{2}$, with equality if and only if $\tilde{\mathbb{H}}=c \mathbb{R}_{s}^{R}$ with arbitrary real $c \neq 0$. Therefore, this choice of $\tilde{\mathbb{H}}$ maximizes $d^{2}$. Setting $c=1 / \sigma_{z}^{4}$, the RDD test statistic (11) is thus obtained as

$$
\bar{T}_{\mathrm{D}}(x)=\left\langle\overline{\mathbb{H}}_{\mathrm{D}} x, x\right\rangle \text { with } \tilde{\mathbb{H}}_{\mathrm{D}}=\frac{1}{\sigma_{z}^{4}} \mathbb{R}_{s}^{\mathrm{R}} .
$$

The RDD allows a formulation and implementation similar to (8). Inserting $\tilde{\mathrm{H}}_{\mathrm{D}}=\sum_{k=-\infty}^{\infty} \sum_{l=0}^{L-1} \sum_{k^{\prime}=k-M_{1}}^{k+M_{2}} \tilde{h}_{k, k^{\prime} ; l}^{\mathrm{D}} \mathbb{P}_{k, k^{\prime} ; l}$ into (12) and using (4), we obtain

$$
\tilde{T}_{\mathrm{D}}(x)=P \sum_{k=-\infty}^{\infty} \sum_{l=0}^{L-1} \sum_{k^{\prime}=k-M_{1}}^{k+M_{2}} \tilde{h}_{k, k^{\prime} ; l}^{\mathrm{D}} \hat{r}_{k, k^{\prime} ; l}^{*}=P \sum_{k=-\infty}^{\infty} \sum_{l=0}^{L-1} \hat{\mathbf{r}}_{k, l}^{H} \mathbf{h}_{k, l}^{\mathrm{D}},
$$

where $\hat{\mathbf{r}}_{k, l}$ is as in (8) and the RF coefficient vector is given by

$$
\mathbf{h}_{k, l}^{\mathrm{D}}=\frac{1}{\sigma_{z}^{4}} \mathbf{r}_{k, l}^{(s)} .
$$

Note that in contrast to the RLD (cf. (9)), no matrix inversions are needed for the design of the RDD.

Comparing (12) with (10), we see that $\tilde{\mathbb{H}}_{\mathrm{D}}$ is simply the orthogonal projection of $\mathbb{H}_{\mathrm{D}}$ onto $\mathscr{R}$, i.e., $\tilde{H}_{\mathrm{D}}=\mathbb{H}_{\mathrm{D}}^{\mathrm{R}}$. If $s[n]$ is an RP with robuston parameters $N, P, M_{1}, M_{2}$, the classical deflection-optimal detector in (10) is equal to the RDD with the same parameters, i.e., $\mathbb{H}_{\mathrm{D}}=\tilde{\mathbb{H}}_{\mathrm{D}}$ and $T_{\mathrm{D}}(x)=\tilde{T}_{\mathrm{D}}(x)$. Hence, the robuston structure of $s[n]$ enforces a robuston structure on $\mathbb{H}_{\mathrm{D}}$. It is important to note that the RDD only presupposes knowledge of the RCs of $s[n]$.

The deflection achieved with the RDD is obtained as $\left\|\mathbb{R}_{s}^{R}\right\|_{H S}^{2} / \sigma_{z}^{4}$, whereas the deflection achieved with the classical deffection-optima detector (10) is $\left\|\mathbb{R}_{s}\right\|_{\mathrm{HS}}^{2} / \sigma_{z}^{4}$. Thus, the loss in deflection resulting from using the RDD is given by $\left(\left\|\mathbb{R}_{S}\right\|_{\mathrm{HS}}^{2}-\left\|\mathbb{R}_{S}^{\mathrm{R}}\right\|_{\mathrm{HS}}^{2}\right) / \sigma_{z}^{4}=$ $\left\|\mathbb{R}_{s}^{\perp}\right\|_{\mathrm{HS}}^{2} / \sigma_{z}^{4}$. Because $\mathbb{R}_{s}^{R}$ is the robuston correlation operator closest to $\mathbb{R}_{s}, \|\left[\mathbb{R}_{s}^{\perp} \|_{\mathrm{HS}}^{2}\right.$ is a measure of how far $s[n]$ is from being an RP.

We finally note that the robust detectors presented in [2] are special cases of the RLD and RDD for $M=1$.

\section{SIGNAL-ADAPTIVE ROBUSTON DETECTORS}

In the previous section, we developed detectors of the likelihoodratio and deflection-optimal types that involve RFs and RCs. According to (9) and (14), the design of the RLD and RDD requires knowledge of the signal RCs $r_{k, k^{\prime} ; I}^{(s)}$. For practical operation, we now propose signal-adaptive versions of the RLD and RDD that incorporate online RC estimation from a single realization of $x[n]$. The only prior knowledge required is the noise variance $\sigma_{z}^{2}$, which in many cases can be reliably estimated during "noise-only" periods.

${ }^{2}$ The inner product of two operators $\mathbb{H}_{1}, H_{2} \in \mathscr{L}$ with kernels $H_{1}\left[n, n^{\prime}\right]$ and $H_{2}\left[n, n^{\prime}\right]$ is defined as $\left\langle\mathbb{H}_{1}: \mathbb{H}_{2}\right\rangle_{\mathrm{HS}} \triangleq \sum_{n=-\infty}^{\infty} \sum_{n^{\prime}=-\infty}^{\infty} H_{1}\left[n, n^{\prime}\right] H_{2}^{*}\left[n, n^{\prime}\right]$. The Hilbert-Schmidt norm is given by $\|\mathbf{H}\|_{\text {HS }}=\sqrt{\langle\mathbf{H I} . \mathbf{H}\rangle_{\mathrm{HS}}}[9]$.

\subsection{Estimation of Robuston Correlations}

Let us first assume that $\mathscr{H}_{1}$ is in force. Here, $\mathbf{R}_{k, l}=\mathbf{R}_{k, l}^{(s)}+\sigma_{z}^{2} \mathbf{I}$, and thus the matrix $\hat{\mathbf{R}}_{k, l}$ given by $\left[\hat{\mathbf{R}}_{k, l}\right]_{m, m^{\prime}}=\hat{r}_{k-M_{1}+m^{\prime}-1 . k-M_{1}+m-1 ; l}$ with $\hat{r}_{k, k^{\prime}: l}$ computed from $x[n]$ according to (4), is an unbiased estimate of $\mathbf{R}_{k, l}^{(s)}+\sigma_{z}^{2} \mathbf{I}$. This suggests to use $\hat{\mathbf{R}}_{k, l}-\sigma_{z}^{2} \mathbf{I}$ as an unbiased estimate of $\mathbf{R}_{k, l}^{(s)}$. However, since $\mathbf{R}_{k, l}^{(s)}$ is always positive definite or semi-definite, a refined estimate is

$$
\hat{\mathbf{R}}_{k, l}^{(s)}=\left[\hat{\mathbf{R}}_{k, l}-\sigma_{z}^{2} \mathbf{I}\right]_{+},
$$

where []$_{+}$denotes the positive (semi-)definite part of a matrix (this can be calculated e.g. by means of an eigendecomposition). By noting that $\mathbf{r}_{k . l}^{(s)}=\mathbf{R}_{k, j}^{(s)} \mathbf{u}$ with the length- $M$ unit vector $\mathbf{u}$ given by $[\mathbf{u}]_{m}=\delta\left[m-1-M_{1}\right]$, an estimate of $\mathbf{r}_{k, l}^{(s)}$ is obtained as $\hat{\mathbf{r}}_{k, l}^{(s)}=\hat{\mathbf{R}}_{k, l}^{(s)} \mathbf{u}$.

Because of the intra-subspace averaging involved in the computation of the $\hat{r}_{k, k^{\prime} ; l}$, the reliability of the estimates $\hat{\mathbf{R}}_{k, l}$ and $\hat{\mathbf{R}}_{k, l}^{(s)}$ (under $\mathscr{H}_{1}$ ) grows with increasing robuston subspace dimension $P$. However, if $\mathscr{H}_{0}$ is in force, $\mathbf{R}_{k J}^{(s)}$ is strongly biased. Fortunately, this bias tends to decrease the resulting adaptive test statistics under $\mathscr{H}_{0}$, which typically does not impair the performance in the sense of causing additional false alams."

\subsection{Signal-Adaptive Online Implementation}

Replacing all RC matrices and vectors in (9) and (14) by their estimates, we obtain estimated RF coefficient vectors of the RLD and RDD as

$$
\hat{\mathbf{h}}_{k, l}^{\mathrm{L}}=\frac{1}{\sigma_{z}^{2}} \hat{\mathbf{R}}_{k, l}^{-1} \hat{\mathbf{r}}_{k, l}^{(s)}, \quad \hat{\mathbf{h}}_{k, l}^{\mathrm{D}}=\frac{1}{\sigma_{z}^{4}} \hat{\mathbf{r}}_{k, l}^{(s)} .
$$

Insertion in (8) and (13) yields the following signal-adaptive versions of the RLD and RDD:

$$
\hat{\bar{T}}_{\mathbf{L}}(x)=P \sum_{k=-\infty}^{\infty} \sum_{l=0}^{L-1} \hat{\mathbf{r}}_{k, l}^{H} \hat{\mathbf{h}}_{k, l}^{\mathrm{L}}, \quad \hat{\mathrm{T}}_{\mathrm{D}}(x)=P \sum_{k=-\infty}^{\infty} \sum_{l=0}^{L-1} \hat{\mathbf{r}}_{k, l}^{H} \hat{\mathbf{h}}_{k, l}^{\mathrm{D}} .
$$

The $(k, l)$ th component of these test statistics can thus be obtained by computing:

1. the LCB coefficients $\alpha_{k, l}^{(p)}=\left\langle x, u_{k, l}^{(p)}\right\rangle, p=0, \ldots, P-1$ by means of an efficient DCT algorithm $[5,6]$;

2. the RC estimates $\hat{r}_{k, k^{\prime}, l}=\frac{1}{p} \sum_{p=0}^{P-1} \alpha_{k, l}^{(p)} \alpha_{k^{\prime}, l}^{(p) *}$ and, in turn, $\hat{\mathbf{R}}_{k, l}, \hat{\mathbf{r}}_{k, l}=\hat{\mathbf{R}}_{k, l} \mathbf{u}, \hat{\mathbf{R}}_{k, l}^{(s)}=\left[\hat{\mathbf{R}}_{k, l}-\sigma_{z}^{2} \mathbf{I}\right]_{+}$, and $\hat{\mathbf{r}}_{k, l}^{(s)}=\hat{\mathbf{R}}_{k, l}^{(s)} \mathbf{u} ;$

3. the RF coefficient vector $\hat{h}_{k, l}^{\mathrm{L}}$ or $\hat{\mathbf{h}}_{k, l}^{\mathrm{D}}$ according to (15);

4. and the vector dot product $\hat{\mathbf{r}}_{k, l}^{H} \hat{\mathbf{h}}_{k, l}^{\mathrm{L}}$ or $\hat{\mathbf{r}}_{k, l}^{H} \hat{\mathbf{h}}_{k, l}^{\mathrm{D}}$.

In fact, steps 3 and 4 can be combined and simplified. For the RLD,

$$
\hat{\mathbf{r}}_{k, l}^{H} \hat{\mathbf{h}}_{k, l}^{\mathrm{L}}=\mathbf{u}^{H} \hat{\mathbf{R}}_{k, l} \frac{1}{\sigma_{z}^{2}} \hat{\mathbf{R}}_{k, l}^{-1} \hat{\mathbf{R}}_{k, l}^{(s)} \mathbf{u}=\frac{1}{\sigma_{z}^{2}} \mathbf{u}^{H} \hat{\mathbf{R}}_{k, l}^{(s)} \mathbf{u}=\frac{1}{\sigma_{z}^{2}} \hat{r}_{k, k, l}^{(s)},
$$

which is simply an estimate of the energy of the $(k, l)$ th robuston $s_{k, l}[n]$ up to a factor. For the RDD,

$$
\hat{\mathbf{r}}_{k, l}^{H} \hat{\mathbf{h}}_{k, l}^{\mathrm{D}}=\frac{1}{\sigma_{z}^{4}} \hat{\mathbf{r}}_{k, l}^{H} \hat{\mathbf{r}}_{k, l}^{(s)},
$$

i.e., steps 3 and 4 merely amount to a length- $M$ dot product. Careful analysis of the computations actually necessary (avoiding repeated calculation of the same RCs $\hat{r}_{k, k}: l$ in the computation of different $\hat{\mathbf{R}}_{k, l}$ ) shows that the overall computational complexity for both detectors is $\mathscr{O}\left(\log N+M^{3} / P\right)$ per signal sample (note that $M$ is typically small). The entire calculation of the test statistic $\hat{T}_{\mathrm{L}}(x)$ 


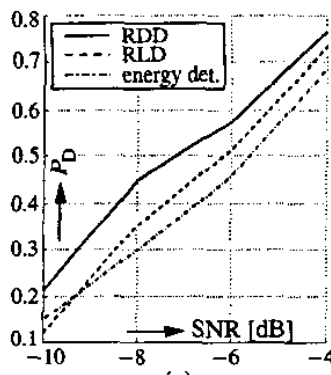

(a)

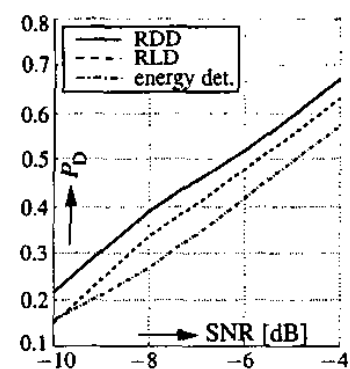

(b)
Figure 2: Power curves at $P_{\mathrm{FA}}=0.01$ of the RLD and $R D D$ (for $N=64, P=8, M_{1}=M_{2}=2$ ) and of the energy detector for $(a)$ the Gaussian process and $(b)$ the Laplacian process.

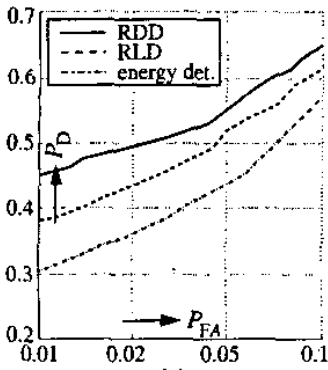

(a)

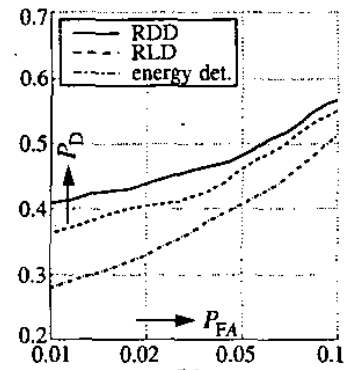

(b)
Figure 3: $R O C$ s at $\mathrm{SNR}=-8 \mathrm{~dB}$ of the $R L D$ and $R D D$ (for $N=64$, $P=8, M_{1}=M_{2}=2$ ) and of the energy detector for (a) the Gaussian process and (b) the Laplacian process.

or $\hat{\bar{T}}_{\mathrm{D}}(x)$ is online because computation of the $(k, l)$ th component $\hat{\mathbf{r}}_{k, l}^{H} \hat{\mathbf{h}}_{k, l}^{\mathrm{L}}$ or $\hat{\mathbf{r}}_{k, l}^{H} \hat{\mathbf{h}}_{k, l}^{\mathrm{D}}$ involves $x[n]$ only within a local time interval (essentially the $k$ th robuston interval $[k N,(k+1) N]$, cf. Fig. 1), and the summation over $k$ in (16) can be performed recursively.

We finally note that the signal-adaptive detectors presented in [2] are special cases of the signal-adaptive RLD and RDD for $M=1$.

\section{SIMULATION RESULTS}

We applied the signal-adaptive RLD and RDD to a nonstationary Gaussian signal process and a nonstationary Laplace-distributed signal process in stationary white Gaussian noise. The signal length was 256 samples. Both signal processes consisted of six timefrequency localized, pairwise mutually correlated random components that were located in the same frequency band and spaced 64 signal samples apart. Monte-Carlo simulations using 5000 realizations were performed to assess the performance of our detectors.

The Laplace-distributed process was constructed as a spherically invariant random process (SIRP) [10]. SIRPs are nonergodic processes that are generated by multiplying a Gaussian process (we used our Gaussian signal process) by an appropriately distributed random amplitude. This results in a Laplacian distribution of each signal sample and a joint second-order distribution (correlation operator) that is equal to that of our Gaussian signal process.

Fig. 2 shows the power curves (i.e., estimated detection probability $P_{D}$ vs. SNR) of the adaptive RLD and RDD for block length $N=64$, subspace dimension $P=8$, and one-sided fitter lengths $M_{1}=M_{2}=2$ at a false alarm probability of $P_{\mathrm{FA}}=0.01$. The power curve of the energy detector [7] is also shown as a performance reference. The SNR was varied by adjusting the noise variance. It is seen that for both types of processes, our detectors perform significantly better than the energy detector, and the RDD performs better than the RLD. The same conclusions can be drawn from the estimated receiver operating characteristics (ROCs)--i.e., $P_{\mathrm{D}}$ vs.

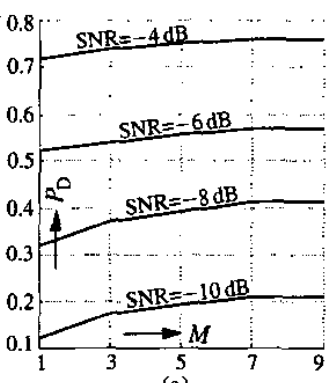

(a)

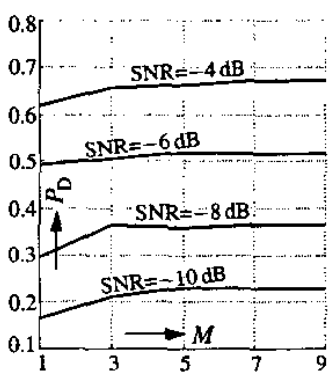

(b)
Figure 4: Detection probability (at $P_{\mathrm{FA}}=0.01$ ) of the $R D D$ with $N=64$ and $P=32$ vs. total filter length $M$ for (a) the Gaussian process and $(b)$ the Laplacian process.

$P_{\mathrm{FA}}$-shown in Fig. 3 for the same robuston parameters $(N=64$, $P=8, M_{1}=M_{2}=2$ ) and an SNR of $-8 \mathrm{~dB}$.

Finally, Fig. 4 shows the dependence of $P_{\mathrm{D}}$ (at $P_{\mathrm{FA}}=0.01$ and various SNRs) of the RDD with $N=64$ and $P=32$ vs. the total filter length $M$ (still for $M_{1}=M_{2}$ ). It is seen that a larger filter length is advantageous up to a certain point (this allows to take advantage of the temporal correlations of the signal). Thus, in this case, the robuston detectors improve on the robust detectors of [2] that are obtained as special cases for $M=1$. However, our experiments showed that this behavior is quite sensitive to the robuston parameters $N, P$ and the properties of the signal.

\section{CONCLUSION}

We presented two robuston methods for signal-adaptive detection of a nonstationary random signal in white Gaussian noise. These detectors use a reduced-detail description of the signal's secondorder statistics that allows the relevant statistics (robuston correlations) to be estimated with enhanced statistical stability. All signal processing is online and based on efficient local cosine basis transformations. Simulation results demonstrated the good performance of our detectors for processes with strong temporal correlations and for an appropriate choice of certain design parameters.

\section{REFERENCES}

[1] F. Hlawatsch, G. Matz, and M. Jachan, "Robuston methods for stable statistical signal processing: Principles and application to nonstationary signal estimation," in Proc. IEEE ICASSP-2003, vol. VI, (Hong Kong), pp. 649-652, April 2003.

[2] G. Matz and A. Raidl, "Robust detection of nonstationary random signals belonging to $p$-point uncertainty classes," in Proc. IEEE ICASSP. 2003, (Hong Kong), pp. 641-644, April 2003.

[3] G. Matz and F. Hawatsch, "Minimax robust nonstationary signal estimation based on a p-point uncertainty model," J. Franklin Inst. vol. 337 , pp. $403-419$, July 2000.

[4] G. Matz, F. Hlawatsch, and A. Raidl, "Signal-adaptive robust timevarying Wiener filters: Best subspace selection and statistical analysis," in Proc. IEEE ICASSP-200I, (Salt Lake City, UT), pp. 39453948, May 2001.

[5] S. G. Mallat, A Waveler Tour of Signal Processing. San Diego; Academic Press, 1998.

[6] http://www-stat.stanford.edu/ wavelab.

[7] H. V. Poor, An Introduction to Signal Detection and Estimation. New York: Springer, 1988.

[8] C. R. Baker, "Optimum guadratic detection of a random vector in Gaussian noise," IEEE Trans. Comm. Technol, vol. 14, pp. 802-805, Dec. 1966.

[9] A. W. Naylor and G. R. Sell, Linear Operator Theory in Engineering and Science. New York: Springer, 2nd ed., 1982.

[10] M. Rangaswamy, D. D. Weiner, and A. Öztürk. "Simulation of correlated non-Gaussian interference for radar signal detection;" in Proc. Asilomar Conf. Signals, Systems, Computers, (Pacific Grove (CA)), pp. 148-152, Nov. 1991 . 